\title{
Rassistische Rechtswissenschaft
}

Ich möchte mich darauf beschränken, auf zwei Erscheinungsformen der Fremdenfeindlichkeit rassistischer Prägung im rechtswissenschaftlichen Bereich hinzuweisen. Sie müssen uns zu denken geben, weil sie sich gegen jüdische Mitbürger richteten, deren Familien jahrhundertelang mit Deutschland verbunden waren. Daß der Rassismus dennoch fortwirkte, zeigt die Schwierigkeiten echter und dauerhafter Integration.

1. Wenn von rassistischer Ausschaltung jüdischer Juristen die Rede ist, muß zunächst an den Nationalsozialismus und in diesem Zusammenhang an Carl Schmitt erinnert werden, der in der Weimarer Zeit die Wissenschaft vom öffentlichen Recht mit vertrat, ohne Juden auszugrenzen. Aber nach 1933 wurde er Vorsitzender der Reichsgruppe Hochschullehrer im Deutschen Rechtswahrerbund. Als solcher leitete er am 3./4. Oktober 1936 eine Tagung über »Die deutsche Rechtswissenschaft im Kampf gegen den jüdischen Geist« und hielt dabei die zusammenfassende Schlußansprache ${ }^{1}$. Darin finden sich etwa folgende Formulierungen:

"Durch alle Referate hindurch zog sich die Erkenntnis, wie stark das jüdische Gesetzesdenken auf sämtlichen Gebieten des Rechtslebens zur Herrschaft gelangte und wie wenig dieses Gesetzesdenken mit dem Rechts- und Gesetzesgefühl des deutschen Menschen auch nur vergleichsweise in Beziehung gebracht werden kann ...

Entscheidend ist ferner das Problem der Zitate. Nach einer solchen Tagung ist es gar nicht mehr möglich, einen jüdischen Autor wie cinen andern Autor zu zitieren. Geradezu unverantwortlich wäre es, einen jüdischen Autor als Kronzeugen oder gar als eine Art Autorität auf einem Gebiet anzuführen. Ein jüdischer Autor hat für uns keine Autorität, auch keine rrein wissenschaftliche< Autorität. Diese Feststellung ist der Ausgangspunkt für die Behandlung der Zitatenfrage. Ein jüdischer Autor ist für uns, wenn er überhaupt zitiert wird, ein jüdischer Autor. Die Beifügung des Wortes und der Bezeichnung sjüdisch` ist keine Äußerlichkeit, sondern etwas Wesentliches, weil wir ja nicht verhindern können, daß sich der jüdische Autor der deutschen Sprache bedient...

Wenn es aus einem sachlichen Grund notwendig ist, jüdische Autoren zu zitieren, dann nur mit dem Zusatz sjüdisch،. Schon von der bloßen Nennung des Worts sjüdisch « wird ein heilsamer Exorzismus ausgehen ...

Das Wichtigste aber, was sich in diesen Tagen für uns als Ergebnis herausgestellt hat, ist doch wohl die klare und endgultige Erkenntnıs, daß jüdische Meinungen in ibrem gedanklichen Inhalt nicht mit Meinungen deutscher oder sonstiger nichtjüdıscher Autoren auf eine Ebene gestellt werden konnen...

Uns beschäftigt der Jude nicht seiner selbst wegen. Was wir suchen und worum wir kämpfen, ist unsere unverfälschte eigene Art, die unversehrte Reinheit unseres deutschen Volkes. >Indem ich mich des Juden erwehre`, sagt unser Führer Adolf Hitler, `kämpfe ich für das Werk des Herrne. (Hervorhebungen im Original gesperrt.)

Es gilt, sich diese »Positionen und Begriffe ${ }^{2}$ vor Augen zu halten, wenn die in den letzten Jahren sämtlich wieder aufgelegten Werke dieses bis heute vielfach in wissenschaftlichen Tagungen Geehrten zitiert und diskutiert werden.

2. Aber wichtiger scheint mir, daß dieses Extremphänomen kein Betriebsunfall der Geschichte gewesen ist, sondern längst vorbereitet war und, wie wir sehen, bis zur Gegenwart fortwirkt. Daher wähle ich als zweites Beispiel das Schicksal von Hugo Preuß, des Schöpfers der Weimarer Verfassung. Preuß war ein glänzender, höchst origineller Jurist, Schüler Otto von Gierkes und bei diesem in Berlin 1889 habilitiert. Aber Preuß war Jude. Wohl auch deshalb erhielt er zunächst keine Professur. Dafür ließ er sich in die Berliner Stadtverordnetenversammlung wählen. Aber auch hier

1 Abgedruckt in: Deutsche Juristen-Zeitung 1936, Sp. $193 \mathrm{ff}$.

2 So der Titel von Carl Schmitts Aufsatzsammlung, 1940 
stieß er auf Schwierigkeiten. Als das preußische Kultusministerium I 898 die Tätigkeit jüdischer Volksschullehrerinnen durch Verwaltungsanweisung auf die Erteilung jüdischen Religionsunterrichts beschränken wollte, sah Preuß den Simultancharakter der Volksschule, die Lehrfreiheit, vor allem aber die kommunale Schulhoheit beeinträchtigt. Er widersprach scharf, auch sarkastisch. Dies führte nicht nur zu antijüdischen Presseäußerungen gegen Preu $\beta$ und spitzte den christlichen-jüdischen Aspekt einer zunächst punktuellen, Preuß vor allem aus kommunalpolitischem Blickwinkel interessierenden Frage zu. Sondern der Oberhofmeister der Kaiserin drückte dem Kultusminister und dem Rektor der Universität in geheimen Schreiben Ihrer Majestät Mißfallen und den Wunsch aus, "Gefahren abzuwenden, daß solche jüdischen Spötter Lehrer unserer heranwachsenden Jugend sind.« Die Sache blieb nicht geheim und erschien als Ausübung persönlicher Macht der Kaiserin problematisch. Dennoch scheint sie wesentlich dazu beigetragen zu haben, Preuß' wissenschaftliche Laufbahn zu blockieren ${ }^{3}$. Erst an der wesentlich von der Kaufmannschaft, unter der viele Juden waren, getragenen Handelshochschule Berlin erhielt Preuß eine Professur.

Nach diesen Erfahrungen veröffentlichte er im Ersten Weltkrieg eine tiefschürfende Streitschrift ${ }^{4}$, in der er für einen Versöhnungsfrieden und für eine volksstaatliche, also demokratische Umgestaltung Deutschlands eintrat. Die Antwort war eine Rezension des bedeutenden Ökonomen Gustav Schmoller's. Auch hier lohnt es, sich einige Auszüge zu vergegenwärtigen:

"Preuß ist einer der begabtesten neueren Staatsrechtslehrer; in gewissem Sinne ein Schüler Gierkes, hat er doch dessen Wendung zu einer mehr konservativen Betrachtung unseres politischen Lebens nicht mitgemacht, sondern im Gegenteil die Genossenschaftsgedanken fortschrittlich ausgedeutet. Er ist einer der Häuptlinge des Berliner kommunalen Freisinns geworden, der, sozial auf semitischer Millionärsbasis beruhend, unsere Hauptstadt mehr oder weniger beherrscht. Und es will mir immer vorkommen, daß in diesen Kreisen, so tüchtig und ehrbar sie sind, der politische Horizont und das politische Urteil doch zu sehr von dem einen Gedanken erfüllt ist: in ihren Kreisen sei eine solche Überlegenheit von Intelligenz, Charakter und Talent, daß es ungerecht für Staat und Gesellschaft sei, daß ihr eng zusammenhaltender Kreis die Universitäten, das Heer, das hohe Beamtentum noch nicht so unbedingt beherrsche, wie das bezüglich der Stadt Berlin und ihrer Verwaltung der Fall sei ...

Aber deshalb ist es doch entfernt nicht allgemein richtig, daß wir die Rechtsgleichheit unserer Verfassung gröblich verletzen, weil einzelne Ämter noch nicht jedem jüdischen ungetauften Bewerber erreichbar sind, weil einzelne Regimenter das ihnen zustehende freie Wahlrecht des Offizierkorps noch zum Judenausschluß benutzen, weil an den Universitäten noch nicht alle zahlreichen jüdischen Privatdozenten so rasch Professoren werden, als sie persönlich glauben, es nach ihrem Talent zu verdienen ...

Wie rasch haben die jüdischen Privatdozenten und Professoren zugenommen! Wie rasch haben die Juden es erreicht, daß an einzelnen Kliniken jahrelang nur jüdische Assistenten angestellt wurden, wie bewahrheitete sich in manchen Fakultäten die Prophezeiung, daß der erste jüdische Ordinarius in zehn Jahren fünf und mehr andere Juden nach sich ziehe! Die Benachteiligung der Juden im preußischen Staatsleben ist heute fast schon dem Verschwinden nahe und macht bereits dem Gegenteil da und dort Platz."

Wir sehen: Diskriminierung, Ausgrenzung begegnet uns schon hier; die Grundlagen für später waren gelegt. Hugo Preuß hatte das Glück, dem Schicksal Walter Rathenaus zu entgehen; er starb 1925 eines natürlichen Todes.

Aber er hat nicht nur die erste demokratische Verfassung für Deutschland entworfen, sondern die Überwindung der Ausgrenzung vorgelebt. Durch beides hat er den

\footnotetext{
3 Dazu und zum folgenden Dian Schefold, Hugo Preuß (1860-1925), in: Deutsche Juristen jüdischer Herkunft, I 993 , S. 429 ff. ( 433 f., 443 f.).

4 Hugo Preuß, Das deutsche Volk und die Politik, igis.

s Schmoller, Obrigkeitsstaat und Volksstaat, ein mißverständlicher Gegensatz, in: Schmollers Jahrbücher Bd. $40 / 2$ (1916), S. $423 \mathrm{ff}$.
} 
Grund heutiger politischer Ordnung gelegt. Wer an die Verbindung der beiden Ele-

mente dieses Lebenswerks rührt, gefährdet beide. Es ist an der Zeit, an diesen Zusammenhang zu erinnern.

Dian Schefold

\section{Die Rassenlehre des Nationalsozialismus als Verneinung des Verfassungsstaates}

In Mölln sind Menschen ermordet worden, nur weil sie "Ausländer« waren. Es mehren sich die Nachrichten, daß Mitbürger beschimpft und bedroht werden, nur weil sie "Juden« sind. Für die Vorverurteilung von Angehörigen der Roma und Sinti genügt, daß sie "Zigeuner « sind. Ausländer, Juden, Zigeuner raus! Solche Ausgrenzungsparolen und ihre furchtbare praktische Umsetzung drängen den Vergleich mit der Rassenlehre des Nationalsozialismus und ihrer millionenfach tödlichen Praxis geradezu auf - beängstigend für die Betroffenen, bedrängend für uns alle. Allerdings darf die unbedingt notwendige Erinnerung an den nationalsozialistischen Rassenwahn nicht zu einer schlichten Gleichsetzung und zu einfachen Parallelen führen. Die Auseinandersetzung mit der Rassenidologie des Nationalsozialismus ist für uns wichtig nicht nur wegen der Parallelen von damals und heute, sondern zumindest ebenso wegen der Unterschiede von damals und heute. Erst wenn wir beide Fragen zusammen stellen, die nach den Parallelen und die nach den Unterschieden, können wir der zentralen Frage nicht ausweichen: Was sind die Gründe der aktuellen Ereignisse in unserer Gesellschaft. Allzu leicht erfüllt sonst die schnelle Rede vom Rassismus und Faschismus eine Verdrängungsfunktion, denn die Rassisten und Faschisten sind natürlich immer die anderen, und dies als »ewig Gestrige«.

Die Rassenlehre des Nationalsozialismus war keine konsistente und wissenschaftlich fundierte Weltanschauung, sondern eine irrational motivierte Feindbildbegründung. Für das völkischrassische Ideengut konnte sie sich ebenso wie für einen aktiven Antisemitismus auf eine lange und breit getragene Tradition berufen. Vor allem der Antisemitismus hatte in Europa eine lange und schlimme Geschichte. Die sich in zahlreichen Vertreibungen und Pogromen austobende Judenfeindschaft des Mittelalters wurde in der 2. Hälfte des i 9 . Jahrhunderts durch den sog. "modernen « Antisemitismus ergänzt oder ersetzt. Ihm lagen in erster Linie wirtschaftliche und soziale Motive zugrunde. ${ }^{1}$ In den die Entwicklung zum Hochkapitalismus begleitenden Umbruchkrisen und dann vor allem in den wirtschaftlichen und politischen Krisenzeiten der Weimarer Republik erfüllte er eine Sündenbockfunktion. "Der >reiche Jude< war kein Mensch aus Fleisch und Blut, sondern ein abstrakter Typus, auf den sich Neid- und Haßgefühle projizierten. "Er stand für alles, was das rechte und rechtsradikale Lager ablehnte: die ungeliebte Republik wurde als »Juden-Republik« beschimpft; zugleich stand »der Jude« für die »bolschewistische Gefahr«.

Der Nationalsozialismus brauchte die vorhandenen Elemente nur auszuweiten und zu intensivieren. Durch die Verbindung der Rassenlehre mit dem von dem Staatsrechtler Carl Schmitt propagierten Freund-Feind-Denken erfuhr der Antisemitismus eine bis dahin unbekannte Brutalisierung. Nur das deutsche Volk bildete die "Volksgemeinschaft", die im Führer ihre existentielle Verkörperung erlebte. Konstitutionsprinzip der "völkischen Einheit« war die rassistisch begründete "völkische Gleichheit «: Nur Angehörige der »arischen Rasse» waren als "Artgleiche " Gemeinschaftsmitglieder; Angehörige "nichtarischer Rassen" (und zu ihnen wurden neben den Juden dann immer weitere Gruppen gezählt: Zigeuner, Polen, überhaupt »Fremdvölkische«) waren »Artfremde« und deshalb »Gemeinschaftsfremde«. Zwischen »Art-

I Z. T. in wörtlicher Anlehnung an D. Majer, Grundlagen des nationalsozialistischen Rechtssystems, 1987, S. $40 \mathrm{ff}$. 